\title{
DEMOCRATIZAR LA DEMOCRACIA: EL PROGRAMA DESCOLONIZADOR DEL COSMOPOLITISMO SUBALTERNO
}

\author{
Democratizing Democracy: \\ the Decolonizing Program of Subaltern Cosmopolitanism \\ Miguel Mandujano Estrada \\ Universidad de La Laguna \\ mmandujano@ull.es
}

\begin{abstract}
Resumen:
En este trabajo presentamos dos formas en las que puede enunciarse un programa descolonizador a partir del cosmopolitismo subalterno, la plataforma de Boaventura de Sousa Santos. Partiendo de la crítica a una modernidad en confluencia con el capitalismo, enmarcamos este programa como una práctica descolonizadora frente a las pulsiones fascistas de la sociedad. Para conseguir este propósito explicaremos la manera en la que la modernidad extendió la desigualdad y la exclusión como mecanismo de integración, estableciendo una racionalidad colonial de límites radicales. El programa descolonizador del cosmopolitismo subalterno representa, frente a este pensamiento colonial, un campo de ampliación e intensificación democrática basada en un principio ético, político y epistemológico fundamental identificado con la ecología de saberes y las epistemologías del Sur.
\end{abstract}

\section{Palabras clave:}

Cosmopolitismo subalterno, descolonización, Boaventura de Sousa Santos, democratización, epistemologías del Sur.

\begin{abstract}
:
In this paper, we present two ways in which a decolonizing program can be enunciated from Subaltern Cosmopolitanism, the platform of Boaventura de Sousa Santos. Starting from the critique of a modernity in confluence with capitalism, we frame this program as a decolonizing practice against the fascist impulses of society. To achieve this purpose, we will explain the way in which modernity extended inequality and exclusion as a mechanism of integration, establishing a colonial rationality with radical borders. The decolonizing program of Subaltern Cosmopolitanism represents, facing this colonial thought, a field of democratic expansion and intensification based on an ethical, political and epistemological fundamental principle identified with the Ecology of Knowledge and the Epistemologies of the South.
\end{abstract}




\section{Keywords:}

Subaltern Cosmopolitanism, Decolonization, Boaventura de Sousa Santos, Democratization, Epistemologies of the South.

Recibido: 29/11/2020

Aceptado: 24/12/2020

\section{INTRODUCCIÓN}

La salud de la democracia en la actualidad es indeterminada. Por un lado, la emergencia de las derechas más radicales, a instancias, en parte, de una suerte de «monarquía del miedo» (Nussbaum, 2019), ha contragolpeado la agitación política de los años posteriores al inicio de la crisis de 2008. Por otra parte, y a pesar de una desafección generalizada por las estructuras participativas, la última década ha sido testigo del surgimiento de nuevas y múltiples formas de expresión y participación; toda una nueva galaxia mediática como forma de acción política conformada por plataformas de escrutinio digital y vigilancia de la democracia así como formas de resistencia y mecanismos de justicia alternativa, indicadores unos y otros de una novel democracia monitorizada (Keane, 2018). No obstante, hemos asistido también durante el último decenio a la consolidación de una variante de la acumulación capitalista basada en la financiarización, la concentración de la riqueza, la explotación intensiva de los recursos naturales y la vulneración de derechos que hace «particularmente insidiosa» la articulación entre capitalismo, colonialismo y patriarcado (Santos, 2016, p. 320), situación que en este texto tomamos como punto de partida. No es que las relaciones del capitalismo con el colonialismo y el patriarcalismo sean exclusivas de su estadio financiero, pues han estado presentes a lo largo de toda su historia bajo la forma de racismo, esclavitud, colonialismo político, paternalismo, machismo, sexismo, violencia sexual, etc., y aunque la confluencia con la democracia amenazó esta vinculación, las partes han respondido con «pulsiones fascistas» que les han permitido reinventarse y persistir. Por otro lado, aunque podríamos decir que estamos asistiendo también a la aparición de una especie de neofascismo (Guamán et al., 2019) o posfascismo -como prefiere Ilamarlo Enzo Traverso (2018) - que permite a las derechas reaccionarias legitimar el autoritarismo y minar los derechos sociales, con pulsiones fascistas nos referimos no a la ideología sino a un régimen de relaciones sociales de poder desiguales en el que la parte más fuerte tiene un poder tan superior sobre el débil que ejerce una suerte de derecho de veto y disposición «sobre sus deseos, necesidades o aspiraciones de vida digna» (Santos, 2016, p. 320).

Desde el cosmopolitismo subalterno, que es como denominamos a la plataforma crítica de Boaventura de Sousa Santos, presentaremos dos formas complementarias que bien pueden representar un programa o propósito programático para hacer frente a 
este fascismo social y, en general, a la etapa actual de la modernidad/capitalismo ${ }^{1}$, expresión esta última con la que señalamos tanto la independencia como la estrecha relación entre ambos procesos históricos y cuyo encuentro inaugura para nuestro autor la crisis de la racionalidad moderna.

Para Santos pues, es la extensión del capitalismo como modo de producción dominante la peculiaridad histórica de la modernidad (1998, p. 90), una confluencia que rompe la dialéctica que mantenían hasta entonces los pilares de la regulación y la emancipación a favor del eje regulatorio, que pasó a regir los procesos de la desigualdad y la exclusión en perjuicio de la igualdad y la inclusión (2005a, p. 195). De esta tensión dialéctica surgen dos formas cognitivas. En primer lugar, el conocimiento-emancipación, entendido como la trayectoria que va desde un estado de ignorancia, Ilamado colonialismo, a un estado de saber, designado como solidaridad. En segundo lugar, el conocimiento-regulación o la trayectoria que va desde un estado de ignorancia, denominado como caos, a un estado de saber, Ilamado orden (2003, p. 87, 2014). En la época postcolonial, no obstante, la dialéctica regulación/emancipación toma la forma de dos movimientos diferenciados: el retorno de lo colonial y el colonizador, por un lado, y el cosmopolitismo subalterno por el otro.

Es decir, el cosmopolitismo subalterno no es solo la pertenencia común de una comunidad de, o la solidaridad entre, las personas oprimidas, sino que es también la forma que en la postcolonialidad toma el eje de la emancipación y su forma cognitiva. Por otro lado, se trata también de la manera en que nos referimos al aparato crítico en la obra de Santos. Este cuerpo está conformado por una epistemología crítica, de donde surge la noción de las epistemologías del Sur como exigencia de justicia epistémica (2014), y por la necesaria renovación del Estado (2005b). La preferencia por la fórmula cosmopolitismo subalterno busca subrayar la práctica sociopolítica, no sin reconocer el principio crítico de una ecología de saberes y las epistemologías del Sur. ${ }^{2}$

En lo siguiente, presentaremos algunas de las consecuencias del rompimiento entre regulación y emancipación que está en el origen de la crisis de la racionalidad moderna. Volveremos luego sobre la idea del fascismo social, poniéndola en relación con la necesidad de una intensificación de la democracia para destacar después el sentido de lo colonial y los términos de una práctica descolonizadora. Finalmente,

\footnotetext{
${ }^{1}$ En alusión al concepto modernidad/colonialidad (Aníbal Quijano, Walter Mignolo y otros/as) que establece los orígenes de la modernidad en la dominación del continente americano y el control europeo del Atlántico y no en la llustración o en la Revolución Industrial.

2 En este sentido, el cosmopolitismo subalterno podría considerarse también una epistemología política, por referirnos al trabajo reciente de Fernando Broncano (2020), una contribución que sintetiza con madurez el trabajo de Miranda Fricker, José Medina, Lindsey McGoey, y otros/as en el ámbito de la justicia e injusticia epistémica. Sobre el trabajo más reciente de Santos en el Centro de Estudios Sociales de la Universidad de Coímbra puede verse el volumen Conocimientos nacidos en las luchas. Construyendo las epistemologías del Sur (Santos y Meneses, 2020).
} 
enunciaremos las intenciones programáticas del cosmopolitismo subalterno que dan título a este ensayo.

\section{LA GESTIÓN MODERNA DE LA DESIGUALDAD Y LA EXCLUSIÓN}

El ejercicio del poder en las relaciones sociales evidencia que estas pueden estructurarse, jerarquizarse y transformarse. Para Santos (2003), estas relaciones sociales de poder funcionan en constelaciones estructurales, un complejo de relaciones domésticas, laborales, económicas, comunitarias, sociales, internacionales, etc., siempre políticas. En este sentido, la transformación de las relaciones sociales de poder exige una refundación de la política y, al mismo tiempo, una transformación de la política comprende una refundación en las relaciones del ejercicio del poder.

Antes afirmamos que la crisis de la modernidad podía ser entendida como el rompimiento entre los pilares de la regulación y la emancipación. De ellos emergen principios fundamentales, como lo son la libertad y la ciudadanía en el caso del pilar emancipatorio y la desigualdad y la exclusión para su contraparte regulatoria. La modernidad, lejos de reconocer estos últimos intentó minimizarlos, estableciendo un proceso de gestión para mantenerlos en niveles tolerables y definir a través de ellos la forma de pertenencia social (Santos, 2006a, p. 259). Dos arquetipos de la modernidad ilustran adecuadamente la crítica a estos procesos: el reconocimiento de que el principio de la integración social en la sociedad capitalista -las relaciones entre capital y trabajo- se basa en la desigualdad entre el capital y la fuerza de trabajo (Marx) y el estudio de los mecanismos de exclusión con los que el poder se apropia de los sujetos (Foucault).

Para Santos (2006a), la desigualdad y la exclusión son sistemas de pertenencia social jerarquizada de los que se forma parte de manera diferenciada y/o opuesta. En el sistema de desigualdad la pertenencia se da por un mecanismo de integración subordinada mientras que en el sistema de exclusión, la pertenencia es determinada por la segregación, como ocurre, por ejemplo, en la clasificación social. Estos sistemas son tipos ideales, ya que en la práctica los grupos se insertan en combinaciones complejas de ambos procedimientos. En el extremo, un sistema de desigualdad puede estar articulado con un sistema de exclusión (el caso de los intocables en el sistema de castas indio es el ejemplo más recurrido). En otro sentido, tanto la desigualdad como la exclusión permiten diferentes grados. El grado extremo de la exclusión es el exterminio. El grado extremo de la desigualdad es la esclavitud (pp. 260-261).

La gestión moderno-capitalista de la desigualdad y la exclusión es un proceso político multidimensional cuya complejidad se agrava en la medida en que aumentan las desigualdades y las exclusiones. La ambivalencia de los sistemas de desigualdad y exclusión se forma a través de un enorme dispositivo de normalización calificador y descalificador que se extienden como principio de jerarquización social. Por ejemplo, la descalificación del sujeto (como inferior, loco, criminal, etc.) consolida una exclusión, pero al mismo tiempo, busca ser traducida a unas reglas jurídicas que puedan definirla y someterla por medio de un dispositivo de normalización. Así pues, en la base de la exclusión subsiste un tipo de pertenencia que se afirma por la no 
pertenencia, una pertenencia por la exclusión, como el loco definido en relación con la calificación de lo cuerdo y el pobre respecto a la posesión de patrimonio.

También el racismo y el sexismo son formas de jerarquización. Se trata, en este caso, de sistemas basados en dispositivos de verdad que realizan una especie de colaboración en rechazo o prohibición de todo lo que cae del otro lado de la línea en la separación radical que hace la racionalidad moderna entre lo integrado y lo excluido. En estas formas de jerarquización, afirma Santos (2006a), se pretende una integración subordinada por la nueva organización del trabajo. En el racismo, el principio de exclusión se basa en la jerarquía de las razas, de manera que la integración desigual ocurre, primeramente, mediante la explotación colonial y después, a través de la inmigración. En el caso del sexismo, el principio de exclusión se basa en la distinción entre el espacio público, el espacio privado y el principio de la integración desigual. Ocurre así, por ejemplo, con el papel que la mujer desempeña en la reproducción de la fuerza de trabajo en la familia, y luego, a través de la integración en formas precarizadas del trabajo (p. 261).

Tanto la racialización como la sexización de la fuerza de trabajo son dispositivos de jerarquización que combinan la desigualdad y la exclusión y muestran la articulación entre capitalismo, colonialismo y patriarcalismo, con el agravante de que en las sociedades sujetas al colonialismo político los principios de la regulación no establecieron una relación dialéctica con la emancipación y quedaron sujetas a la violencia de la coerción o a la violencia de la asimilación (Santos, 2010).

Ahora bien, la regulación social en la modernidad capitalista está constituida por estos procesos que generan desigualdad y exclusión, pero al mismo tiempo, establece otros mecanismos que puedan controlar o mantener estos procesos dentro de ciertos límites. No hay duda de que el Estado capitalista acepta el desafío de mantener la cohesión social en una sociedad atravesada por los sistemas de desigualdad y exclusión. En el primer caso, a través de mecanismos como los derechos sociales y económicos universales, las rentas mínimas y de inserción y las políticas compensatorias. En cuanto a la exclusión, la función del Estado consiste en distinguir aquellas formas que deben ser objeto de asimilación o de segregación: la distinción entre el loco peligroso y el que no lo es, entre el buen y el mal inmigrante, entre el pueblo indígena bárbaro o el asimilable, entre el comportamiento sexual tolerable y el intolerable, etc. (Santos, 1995, 2006a, p. 264)

\section{FASCISMO SOCIAL E INTENSIDAD DEMOCRÁTICA}

La paradoja consiste en que la gestión moderna de la desigualdad y la exclusión supuso su control mediante estas tecnologías de integración jerarquizada que, en realidad, las extienden y alientan. Incluso el Estado social del bienestar ha terminado por sucumbir ante las reinvenciones capitalistas, en concreto, a la forma neoliberal del capitalismo financiero y a las pulsiones fascistas con la que sus actores reaccionan ante la transformación progresista. Como antes apuntamos, con fascismo social no nos referimos a una postura ideológico-política sino a un régimen social y civilizatorio que sacrifica la democracia ante las exigencias del capitalismo y fomenta ese sacrificio bajo distintas formas de sociabilidad fascista. Santos enuncia estas formas de sociabilidad en «Reinventar la democracia» (2005b, 2005a), y aunque podríamos 
reunirlas en la expresión «pulsiones fascistas» (2016, p. 320), las distinciones que hace nuestro autor en «Reinventar...» colocan la emergencia del fascismo social en el marco de un análisis epistémico-crítico (y epistémico-político) que da cuenta de la crisis del contrato social moderno y propone una renovación del Estado bajo la forma del Estado como movimiento social. En nuestro entender, ese análisis sigue siendo relevante como fundamento de la necesaria transformación de las relaciones sociales de poder y el programa del cosmopolitismo subalterno para conseguirlo, sobre todo, cuando el fascismo político parece estar reagrupándose en los embates de la derecha contemporánea.

Así pues, podemos reconocer un fascismo del apartheid social, «la segregación social de los excluidos dentro de una cartografía urbana dividida en zonas salvajes y zonas civilizadas» del que la gentrificación es un ejemplo elocuente (Santos, 2005b, p. 30). Un fascismo del Estado paralelo que utiliza formas de acción estatal distintas a las del derecho positivo y que, además, designa formas protectoras para las zonas civilizadas y formas predadoras para las salvajes. Un fascismo paraestatal que resulta de la usurpación de las prerrogativas estatales de la coerción y la regulación social. Dentro de este último, podemos distinguir un fascismo contractual que se da, por ejemplo, ante la privatización o sustitución de un contrato de servicios públicos por un contrato de consumo o la de un contrato de trabajo por un contrato civil, y el fascismo territorial, una especie de colonización del territorio privado que se da cuando los actores con patrimonio controlan y ejercen su propia regulación en el territorio que ocupan. Podemos referirnos también a un fascismo populista que democratiza «aquello que en la sociedad capitalista no puede ser democratizado» (Santos, 2005b, p. 32), como la transparencia política de la relación entre representantes y representados, un fascismo de la inseguridad, por ejemplo en el lugar de trabajo, y un fascismo financiero.

Este fascismo social ilustra la crisis del contrato social moderno y se agudiza con la consolidación de la variante democrática minimalista en la segunda parte del siglo XX. Esta privilegia el procedimiento de toma de decisiones (Kelsen, Schumpeter) sobre otras dimensiones de la democracia, el tratamiento del pluralismo como forma de incorporación partidaria y formación de las mayorías (Schumpeter, Bobbio), el establecimiento de la burocracia (Weber) y resuelve el problema de la participación a través de la discusión de las escalas y la complejidad (Dahl) (Santos y Avritzer, 2004). No obstante, la representación, aun si se admite que puede facilitar la autorización, no responde a otras dimensiones del problema, como son la dificultad para representar agendas e identidades específicas y la rendición de cuentas.

En el fondo, se trata de un problema de empuje democrático, por eso serán las concepciones no hegemónicas que vinculan la democracia con una forma de vida (formas de democracia directa, comunitaria, de democracia asamblearia, etc.) las que propondrán en el mismo periodo criterios distintos para el reconocimiento de la pluralidad, la participación, la autorización, etc.: una nueva gramática sociocultural y una innovación social institucional de alta intensidad democrática. 


\section{UNA PRÁCTICA DESCOLONIZADORA}

La tensión entre la regulación y la emancipación social cuyo rompimiento encontramos en la base de la crisis del paradigma de la modernidad, funciona de manera diferente para las sociedades metropolitanas y los territorios coloniales. En estos últimos, la dialéctica regulación-emancipación no se aplica por igual a colonos y colonizadores. En primer lugar, la racionalidad moderna establece «un sistema de divisiones visibles e invisibles [...] a través de líneas radicales» epistemológicas, políticas y socioculturales (Santos, 2010, pp. 11-12). En segundo sitio, el establecimiento de estas líneas abisales o abismales impide la relación regulaciónemancipación y la sustituye por la forma apropiación/violencia, condenando a los sujetos coloniales a un sitio indeterminado entre la violencia de la coerción y la violencia de la asimilación, como afirmamos más arriba. La universalidad de la tensión regulación-emancipación no compromete su universalidad al ser aplicada del otro lado de la línea a través de la incorporación, la cooptación y la asimilación.

Lo mismo puede decirse de otras constelaciones de relaciones sociales de poder, como la del conocimiento, en la que la ciencia y el derecho detentan el monopolio de la distinción de lo universal, lo verdadero o lo legal, en detrimento de otros cuerpos de conocimiento condenados al epistemicidio (Santos, 2010, 2014). La identificación de esta ciencia con la modernidad, por otra parte, es representativa del dominio del conocimiento-regulación en el que el sujeto es una categoría referida únicamente a él -la parte portadora de la razón- y el objeto es una entidad externa, lo totalmente diferente -la naturaleza-, de manera que la unión entre uno y otro se torna una relación imposible. Además, como afirmara Aníbal Quijano (1992), el carácter de la individualidad moderna radicaliza este contraste, provocando la ausencia del otro y anulando la intersubjetividad (pp. 441-442). Pensemos tan solo en los colonizadores del Nuevo Mundo, que negando la palabra y el estatus de humanidad al indígena configuraron una no-Europa primitiva, objeto de conocimiento de una Europa racional, civilizada y de pleno derecho cognoscitivo (Quijano, 2000). De la misma manera, el conocimiento hegemónico estableció la jerarquización y los procesos que condenan al silencio a los saberes no-hegemónicos como una prolongación cultural y epistémica del genocidio y el colonialismo político.

Volviendo a las formas cognitivas surgidas de la ruptura de la tensión dialéctica entre regulación y emancipación, podemos afirmar que la regulación del recorrido que va de la ignorancia al saber en las relaciones entre sujetos como un camino del caos al orden, dio paso al establecimiento de un mono-criterio de jerarquización epistémica. La identificación del conocimiento-regulación con la ciencia moderna desencadenó, igualmente, otras fracturas (entre el sujeto y el objeto, entre la expectativa y la experiencia, etc.), y solo una segunda ruptura podrá conducirnos a un paradigma prudente en lo epistémico y decente en lo social, bajo la forma de un conocimiento-emancipación que conduzca del colonialismo a la solidaridad (Santos, 2003, pp. 82-87).

Lo colonial representa, en su constitución moderna, no solo lo no legal o lo ilegal, sino lo sin ley (Santos, 2010, p. 16, 2019a); una suerte de estado de naturaleza donde las instituciones de la sociedad no tienen lugar y se originan las líneas abisales en la imposición de un canon que persigue, en términos generales, los fines e invenciones 
del colonialismo imperial. En este, el descubridor se legitima como una especie de creador que establece diferencias entre él mismo y lo descubierto, creando una brecha entre su propia experiencia y la ajena. El descubrimiento es recíproco, es verdad, pero siendo el descubrimiento una relación de poder y saber en sí misma, coloca en una posición de privilegio a quien ostenta más poder. Estas líneas son persistentes y solo el cumplimiento de algunas condiciones podrá dar lugar a un pensamiento postabisal: la copresencia, el reconocimiento de la diversidad del mundo y la relación entre saberes e ignorancias.

Ahora bien, la ecología de los saberes no concibe los conocimientos en abstracto sino como prácticas sociopolíticas que permiten o impiden ciertas intervenciones en el mundo real, por ello es necesario revalorizar las operaciones concretas en la sociedad de acuerdo con la naturaleza de los diferentes conocimientos. Se erige así un principio jerárquico de precaución en el que la preferencia debe ser dada a la forma de conocimiento que garantice mayor participación a los grupos sociales involucrados a través de la traducción intercultural, de manera que se pueda evitar que sea más importante vigilar las fronteras de los saberes relevantes que argumentar sobre sus diferencias internas, en otras palabras, para identificar preocupaciones comunes, enfoques complementarios y/o contradicciones intratables (Santos, 2010, p. 39).

\section{EL PROGRAMA DEL COSMOPOLITISMO SUBALTERNO Y LAS EPISTEMOLOGÍAS DEL SUR}

Hay dos formas en las que podemos enunciar las intenciones programáticas del cosmopolitismo subalterno en la obra de Santos. La primera proviene de un periodo que en español compendian los ensayos de El milenio huérfano (2005a) en los que nuestro autor propone un triple camino: del monoculturalismo a la ecología de saberes, del conocimiento especializado a un conocimiento edificante y de la acción conformista a la acción rebelde. La segunda está sintetizada en La difícil democracia (2016) donde se marca también un triple objetivo: desmercantilizar, democratizar, descolonizar.

El primero de los trayectos remite al dominio de la ciencia moderna y la destrucción de las formas de conocimiento de los pueblos sometidos bajo el colonialismo. En este marco, Santos formula la expansión del presente negado como la clave para evitar el desperdicio de la experiencia (2005a). En otras palabras, reducir la unidad totalizante de la razón moderna y hacerla coexistir con otras racionalidades, asumiendo que cualquier totalidad está siempre compuesta de cierta heterogeneidad a la que sus partes le pertenecen de manera precaria. El instrumento propuesto es una ecología de saberes, capaz de otorgar reconocimiento a las prácticas cognitivas de las clases, pueblos y grupos sociales oprimidos, contrarrestar el arquetipo moderno de conocimiento y establecer las condiciones de posibilidad del diálogo entre conocimientos diversos (pp. 162-167). En el mismo orden de ideas, una ecología de saberes podrá romper también con el escalamiento, esto es, la clasificación que determina qué es lo universal y que instituye, en consecuencia, la irrelevancia de cualquier otra escala posible. Finalmente, el cosmopolitismo subalterno se propone una pluralidad de proyectos colectivos que no se centren en el debate sobre el orden de la forma cognitiva conocimiento-regulación y no sean articulados jerárquicamente. 
La segunda enunciación, utilizando la fórmula «desmercantilizar, democratizar, descolonizar», supone el desafío de reconsiderar profundamente los ejes de la relación entre capitalismo, colonialismo y patriarcado (Santos, 2016). Des-pensar «la naturalización del capitalismo» mediante la acción de una economía en otros términos, des-pensar la naturalización de una democracia de baja intensidad, reconociendo la diversidad democrática del mundo, des-pensar el racismo y el sexismo y señalarlo donde se encuentre.

Desmercantilizar «significa, sobre todo, impedir que la economía de mercado amplíe su radio de alcance hasta transformar la sociedad en una sociedad de mercado» (p. 242). "Democratizar significa des-pensar la naturalización de la democracia liberal representativa y legitimar otras formas de deliberación democrática» (p. 242). Descolonizar significa des-pensar «el racismo justificado como resultado de la inferioridad de ciertas razas o grupos étnicos y no como su causa» ( $p$. 243). Se trata de un proyecto que otorga credibilidad a las nuevas formas de relación entre la tierra y la producción, que reconoce la demodiversidad o diversidad democrática y la búsqueda de nuevas conexiones entre distintos modelos democráticos, que denuncia los principios y estructuras que reproducen el sexismo y el racismo.

Desmercantilizar, democratizar, descolonizar es, asimismo, des-pensar las naturalizaciones en todos los ámbitos de las constelaciones sociales del poder: la familia, el lugar de trabajo, la economía, la escuela, la comunidad..., ampliar la disposición político-epistémica y transformarlas. "Desmercantilizar, democratizar y descolonizar significan, en última instancia, refundar el concepto de justicia social incluyendo en la igualdad y en la libertad el reconocimiento de las diferencias [...], la justicia cognitiva [...] y la justicia histórica» (p. 243).

Finalmente, ambos proyectos hacen necesaria una traducción intercultural e interpolítica mediante la que las aspiraciones y las prácticas de los grupos sociales se hagan mutuamente inteligibles (Santos, 2003). Su complejidad radica en la convivencia entre el reconocimiento de la diversidad y las premisas abisales que insisten (y persisten) en la monoculturalización de los principios. Por otro lado, la traducción intercultural es una teoría-crítica, y por lo tanto, una reflexión hermenéutica que debe partir de la valoración de sí mismo. Frente a un interculturalismo liberal -el interculturalismo de un diálogo sin incidencia para la asimetría cultural- una traducción intercultural crítica debe comenzar por la supresión de las estructuras asimétricas, preguntándose en primer lugar por las condiciones mismas del diálogo (Tubino, 2004, p. 6) y la propia posición como participante en la conversación. Puede parecer de Perogrullo pero el diálogo intercultural, en este sentido, implica no solo un talante epistemológico sino una disposición moral fundamental y un compromiso con la realidad que vincule la práctica con la solidaridad.

Un ejemplo de traducción intercultural, en el sentido anterior, es el de la Universidad Popular de los Movimientos Sociales (UPMS), un diálogo no relativista entre conocimientos que funciona como plataforma de estrategias concretas para construir una sociedad más justa y democrática a través del conocimiento recíproco entre movimientos y organizaciones sociales (Santos, 2006b, 2007, 2019a). 
Dos características de la UPMS Ilaman nuestra atención de manera particular. En primer lugar, que su naturaleza ecológica le exige un ambiente de proximidad y protagonismo en la creación de conocimiento, más conversacional que dialógico, si se nos permite, por evitar las premisas racionalistas del dia-logos. En segundo lugar, la polifonía necesaria en una contrauniversidad pluriversal y subversiva (Santos, $2019 b$ ) de conocimiento recíproco y compartido. Resulta paradójico que el ejemplo de la UPMS lleve el nombre de universidad, una institución que no es, precisamente, un dechado de diálogo o diversidad, casi lo contrario, dominada por un sentido unívoco de conocimiento y al servicio de un modo bastante unitario de vida. De aquí la importancia de interpretar universidad como pluriversidad y subversidad, a ejemplo no solo de la UPMS sino de otras experiencias virtuosas, como la de las universidades indígenas (Huanca, 2017).

\section{CONCLUSIONES}

La transformación de las relaciones sociales de poder pasa menos por las posiciones sobre su existencia que por la amplitud de su ejercicio compartido. Es por ello que democratizar la democracia es fundamentalmente un asunto de intensificación y las democracias de alta intensidad son aquellas en las que el ámbito de poder compartido es amplio, imaginativo y abierto. Asimismo, un ámbito de esta naturaleza es mucho más propicio a desnaturalizar la mercantilización del capitalismo financiero y las relaciones coloniales racistas y/o sexistas. Por otro lado, la ampliación e intensificación democráticas se sustentan en una disposición ético-epistemológica que reconoce los conocimientos y saberes de las personas oprimidas por el capitalismo, el colonialismo y el patriarcado, una ecología de saberes pluriversal y subversiva que es la premisa de una justicia cognitiva y, por extensión, de una justicia social.

La revitalización del fascismo ideológico, en un contexto fundamentalmente democrático, nos sorprende; por ello, la ampliación de los derechos económicos y sociales debe ser una de las tareas que el conocimiento jurídico, social y humanístico establezca como prioritaria, con una postura ecológica, transgresiva incluso, abierta a la imaginación y creatividad democráticas, atenta a las pulsiones fascistas y a las brechas abisales de la racionalidad colonial y a través de un diálogo hermenéutico crítico interpluricultural.

\section{REFERENCIAS}

Broncano, F. (2020). Conocimiento expropiado. Epistemología política en una democracia radical. Ediciones Akal.

Guamán, A., Aragoneses, A., y Martín, S. (Eds.). (2019). Neofascismo. La bestia neoliberal. Siglo XXI de España Editores.

Huanca Soto, R. (2017). Conocimiento y pluriversidad de mundos en universidades indígenas: Amawtay Wasi (Ecuador) y UAIIN-CRIC (Colombia). Estudios Artísticos: Revista de Investigación Creadora, 3(3). https://doi.org/10.14483/25009311.12533

Keane, J. (2018). Vida y muerte de la democracia. Fondo de Cultura Economica. 
Nussbaum, M. (2019). La monarquía del miedo. Una mirada filosófica a la crisis política actual. Ediciones Paidós.

Quijano, A. (1992). Colonialidad y modernidad-racionalidad. En H. Bonilla (Ed.), Los conquistados: 1492 y la población indígena de América (pp. 437-447). Tercer Mundo-FLACSO.

Quijano, A. (2000). Colonialidad del poder y clasificación social. Journal of WorldSystems Research, 6(2), 342-386.

Santos, B. (1995). Toward a New Common Sense: Law, Science and Politics in the Paradigmatic Transition. Routledge.

Santos, B. (1998). De la mano de Alicia. Lo social y lo político en la postmodernidad. Uniandes-Siglo del Hombre Editores.

Santos, B. (2003). Crítica de la razón indolente. Contra el desperdicio de la experiencia. Desclée de Brouwer.

Santos, B. (2005a). El milenio huérfano. Ensayos para una nueva cultura política. Trotta.

Santos, B. (2005b). Reinventar la democracia. Reinventar el Estado. CLACSO.

Santos, B. (2006a). A gramática do tempo. Para uma nova cultura política. Cortez.

Santos, B. (2006b). La universidad popular del siglo XXI. Programa de estudios sobre Democracia y Transformación Global-UNMSN.

Santos, B. (2007). El Foro Social Mundial y el autoaprendizaje: la Universidad Popular de los Movimientos Sociales. Theomai. Estudios Sobre Sociedad y Desarrollo, 15, 101-106.

Santos, B. (2010). Para descolonizar Occidente: más allá del pensamiento abismal. CLACSO-Prometeo Libros.

Santos, B. (2014). Epistemologies of the South. Justice Against Epistemicide. Paradigm Publishers.

Santos, B. (2016). La difícil democracia. Una mirada desde la periferia europea. Akal.

Santos, B. (2019a). El fin del imperio cognitivo. La afirmación de las epistemologías del Sur. Editorial Trotta.

Santos, B. (2019b). Hacia una universidad polifónica y comprometida: pluriversidad y subdiversidad. En Educación para otro mundo posible. CLACSO.

Santos, B. , y Avritzer, L. (2004). Para ampliar el canon democrático. En Democratizar la democracia. Los caminos de la democracia participativa (pp. 35-74). FCE.

Santos, B. , y Meneses, M. P. (Eds.). (2020). Conocimientos nacidos en las luchas. Construyendo las epistemologías del Sur. Ediciones Akal.

Traverso, E. (2018). Las nuevas caras de la derecha. Conversacinoes con Régis Meyran. Siglo Veintiuno Editores Argentina. 
Tubino, F. (2004). Del interculturalismo funcional al interculturalismo crítico. En M. Samaniego y C. G. Garbarini (Eds.), Rostros y fronteras de la identidad (pp. 151164). UCT. 